\title{
La educación emocional en el contexto escolar con alumnado de distintos países
}

\author{
Ana M. Peña y $\mathrm{M}^{\mathrm{a}}$ Carmen Canga \\ Universidad de Oviedo (España)
}

\begin{abstract}
En este artículo se aportan datos descriptivos de los resultados de un estudio sobre necesidades educativas emocionales en sujetos escolarizados en Centros Públicos de la Autonomía de Asturias. La muestra está constituida por 104 sujetos de $6^{\circ}$ Curso de Educación Primaria de los cuales 78 son españoles y 26 inmigrantes de distintas nacionalidades y diferentes culturas. Las aportaciones del estudio se dirigen hacia: a) La identificación de sujetos con carencias emocionales; b) La valoración global del grupoclase; c) Comprobar si se dan diferencias significativas entre grupos de sujetos de una misma edad y en un mismo contexto educativo. Los resultados que se desprenden de las dimensiones que mide el cuestionario "Educación Emocional", facilitan la identificación de los sujetos con necesidades emocionales, proporcionan una visión global de grupoclase y permiten conocer si existen diferencias estadísticamente significativas entre los grupos (españoles e inmigrantes) y el género (varones y mujeres).
\end{abstract}

Palabras clave: Educación emocional, dimensiones emocionales, valoración de necesidades emocionales, culturas diferentes.

The emotional educational in the context school with subjects of different countries. In this article it is proposed some of the results of a study on the educational emotional needs of subjects enrolled in school in Public Centers of the Autonomous Community of Asturias. The sample is constituted by 104 subjects of 6th Course of Primary Education, of which 78 are Spanish and 26 emigrants of different nationalities and different cultures. The contribution of the research go towards: a) The identification of subjects with emotional lacks; b) The assessment of the group class; c) To verify if they exist significant differences, in emotional needs, between Spanish subjects and emigrants of the same age and in the same educational context. The results following from the dimensions measuring the questionnaire "Emotional Education", they provide the identification of the subjects with emotional needs, to have a knowledge general of group-classroom and if exist the significant statistics differences between groups (Spanish and emigrants) and sex (men and women).

Key words: Emotional education, emotional dimensions, valuation of emotional needs, different cultures.

Correspondencia: Ana María Peña del Agua. Área de Métodos de Investigación y Diagnóstico en Educación. Facultad de Ciencias de la Educación. Universidad de Oviedo. C/ Aniceto Sela, s/n. 33005 Oviedo. E-mail: delagua@ uniovi.es 
La llegada de inmigrantes a nuestros centros educativos ha abierto un amplio debate en el que la comunidad educativa no puede permanecer al margen. Hoy, más que nunca, necesitamos de una educación que nos enseñe a aprender a convivir con personas de diferentes países, que nos permita desarrollar nuestra empatía con sujetos de distintas creencias y costumbres, que nos enseñe a ser críticos y a construir sociedades en las que quepan ciudadanos de todas las culturas. No obstante, somos conscientes de que la convivencia entre personas de diferentes países conlleva un largo proceso que supone no solamente estar juntos sino un tipo de acción interactiva y productiva. Exige el contacto e intercambio de opiniones entre los sujetos de una misma sociedad, sean o no inmigrantes. Ello supone un enriquecimiento para el profesorado y alumnado, tanto nativos como de otras realidades y ofrece un cauce para el ejercicio de la tolerancia propiciando relaciones que auguren una buena convivencia.

La escuela, como institución social que es, debe cumplir la importante tarea de integrar en su seno diferentes costumbres, creencias y valores, para lograr, mediante la instrucción, una mejor convivencia entre todos sus componentes. Para Moreno (2006) la escuela, como lugar de encuentro de personas distintas y con intereses no siempre convergentes, debe convertirse en lugar idóneo para que los alumnos aprendan las actitudes básicas de un convivir libremente como son: la democracia, la solidaridad y la participación. Según el Informe Delors (1996) aprender a vivir juntos es habilitar al individuo para convivir en contextos de igualdad, tomando conciencia de las semejanzas y diferencias que existen entre los humanos. Considerando lo anterior, entendemos la mediación intercultural, desde la perspectiva educativa, como medio de integración social que favorece la interacción cultural en condiciones de igualdad y la convivencia entre ciudadanos de origen diferente.

Desde el ámbito de la educación, tanto docentes como pedagogos, psicólogos y profesionales de la educación en general, han puesto el acento en estudiar el cómo escolarizar a los niños y niñas procedentes de otros países, culturas, costumbres y religiones, por múltiples criterios y motivaciones. Pero poco se ha elaborado con relación a los aspectos emocionales y su relación con el aprendizaje social. Sin embargo, la educación de las emociones es, sin duda, un exponente excelente para el desarrollo de las relaciones personales y, por ende, de la convivencia entre distintos grupos. A través de la educación de las emociones, los alumnos pueden conseguir mejorar sus relaciones sociales tanto en el ámbito escolar como en el familiar y ambiental, ya que la competencia emocional influye en todos los ámbitos de la vida y en todas las personas, ya sean o no de distintos países y culturas. Por ello, la educación emocional debería ocupar una parte importante del currículo escolar regulado a través de la normativa que se establezca en cada Comunidad Autónoma. Aprender a vivir de manera democrática y solidaria es el encargo más importante que las sociedades modernas encomiendan a los 
sistemas educativos, de ahí la importancia de la colaboración de todos los implicados en la tarea educativa (Romero, 2008).

Tradicionalmente la escuela se ha centrando en aspectos cognitivos, dejando olvidadas las capacidades emocionales. Sin embargo, las aportaciones científicas actuales destacan la vinculación entre las emociones y el pensamiento como base de toda actividad humana, independientemente de la cultura o país de procedencia de las personas. Desde esta perspectiva transcribimos la opinión de Kirchner (1998:183) que manifiesta lo siguiente:

"aprender en la escuela es todavía en muchas clases un puro aprendizaje mental. Las emociones son más bien algo a lo que no se da importancia. Pero la realidad va introduciéndose de manera creciente en los centros educativos. A la vista de los déficits emocionales y sociales con los que los niños llegan a la escuela, los centros de enseñanza no pueden seguir limitándose por mucho más tiempo a hacer de exclusivos transmisores de conocimientos. Si el objetivo de la escuela es preparar para la vida, deberá contribuir al desarrollo de toda la personalidad de los alumnos".

Considerando lo anterior, y de acuerdo con Collel (2003), creemos necesario que se lleve a cabo una profunda reflexión sobre la importancia de los sentimientos y las emociones, así como la conveniencia de realizar un replanteamiento del tema en el ámbito educativo, de manera precisa y comprometida con la acción, teniendo presente que el desarrollo de las emociones de los niños y la comprensión de las mismas, es un proceso continuo y gradual de aprendizaje que va de las emociones simples a las más complejas (Dueñas, 2002).

La Constitución Española, en el artículo 27, establece que "el objetivo de la educación es el pleno desarrollo de la personalidad humana en el respeto a los principios democráticos de convivencia y a los derechos y libertades fundamentales". El Informe Delors (1996) considera que la educación emocional es un complemento indispensable en el desarrollo cognitivo y una herramienta fundamental de prevención, ya que muchos problemas tienen su origen en el ámbito emocional.

Entre los fines generales formulados en la Ley Orgánica 2/2006, de 3 de mayo, de Educación para las diferentes etapas educativas, aparece como propuesta firme que se tenga en cuenta "el pleno desarrollo de la personalidad y de las capacidades afectivas del alumnado...". Del mismo modo, entre los objetivos planteados en la LOE, encontramos que "se atenderá progresivamente al desarrollo afectivo..." (art. 13.3). De la normativa vigente se desprende un reto que debe ser afrontado por los centros educativos, concretamente por los profesionales de la educación (tutores, profesores, orientadores, etc.), que con o sin formación suficiente y sin herramientas apropiadas, en el tema que nos ocupa, han de afrontar la educación emocional de los alumnos. Son estos profesionales los que han de actuar desde perspectivas más amplias que lo puramente 
académico; han de abarcar otras dimensiones como es el caso de lo afectivo-emocional, componente fundamental en el equilibrio de la persona.

La escuela ha de ser la primera institución en afrontar el reto que conlleva a la comprensión de la autorrealización de cada niño, adolescente o joven, que abarca desde la protección de sus sentimientos, emociones, autoestima, hasta la ampliación de sus oportunidades de elegir, requiere un reconocimiento de su singularidad, de su identidad colectiva y de su personalidad individual, de su particularidad cultural como elemento constitutivo y su derecho a integrarse en la sociedad global con independencia de su origen, cultura y/o creencias, que es algo a lo que no se debe renunciar (Fernández, 2008).

El objetivo primordial de este estudio está orientado a identificar, de manera precisa, las necesidades emocionales que manifiesten los alumnos y alumnas de una misma edad en un mismo contexto educativo y comprobar si existen diferencias significativas entre alumnos españoles e inmigrantes. Más concretamente, qué dimensión o dimensiones (emociones, autoestima, habilidades sociales y habilidades para la vida) son en las que los sujetos manifiestan carencias y que supuestamente puedan influir en conductas apropiadas, dentro y fuera del aula. Además, se trata de ver si existen diferencias significativas desde una perspectiva de género. Las puntuaciones que cada sujeto alcance en cada una de las dimensiones, puede ser el punto de partida para diferenciar al alumnado que necesiten de una evaluación diagnóstica que ayude, mediante una recogida de información más amplia y precisa, a orientar la intervención, así como a diseñar y desarrollar aquellos programas educativos más convenientes, que permitan a los sujetos conseguir una estabilidad emocional apropiada a la edad, una mejor integración en el grupo clase y en la sociedad en general, es decir, a aprender cómo se debe de convivir en una sociedad pluralista y compleja.

\section{METODO}

\section{Diseño}

El tipo de investigación de este estudio es descriptivo, debido a que está orientado al conocimiento de la realidad en un tiempo y en un espacio concreto. El método empleado es descriptivo-comparativo, pues se efectúan análisis descriptivos (media aritmética y desviación típica) para conocer las necesidades emocionales de los sujetos y conseguir una visión general del grupo-clase. También se realiza análisis de varianza (prueba $\mathrm{F}$ de Fisher) para determinar la existencia de diferencias estadísticamente significativas en función de los grupos establecidos y del género. 


\section{Sujetos}

Para la obtención de los datos se tomó una muestra al azar entre los posibles Centros Públicos de Asturias, en los que había matriculados alumnos de diferentes países. Han participado un total de 104 sujetos, pertenecientes a $6^{\circ}$ de Educación Primaria, de los cuales 78 son españoles y 26 inmigrantes. Nos hemos centrado en ese curso por parecernos importante la identificación de necesidades de carácter emocional en sujetos que están próximos a realizar un cambio de Etapa Educativa.

La población emigrante en edad escolar en la citada Autonomía es escasa y más aún en el curso elegido para el estudio. Para este trabajo únicamente se han seleccionado centros públicos, por ser los que tienen mayor número de sujetos matriculados procedentes de otros países, asignados por la Administración Educativa. Los lugares de procedencia de los sujetos del estudio son: Latinoamérica (Colombia, Venezuela, Ecuador y Argentina), Marruecos y China. La figura 1, refleja la distribución de la muestra por grupos de sujetos españoles e inmigrantes.

Figura 1. Distribución de la muestra por grupos de sujetos participantes

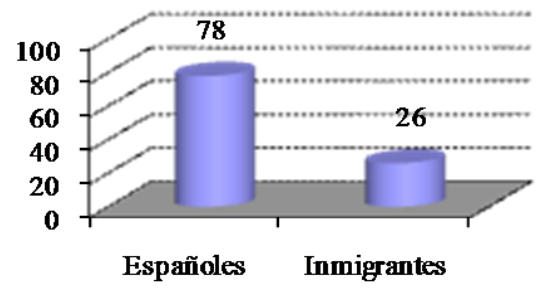

\section{Instrumentos}

Para llevar a cabo el estudio se ha optado por el Cuestionario de Educación Emocional Reducido (CCE-R), elaborado por El GROP (Grup de Recerca en Orientación Psicopedagógica) e incorporado en Álvarez González (2001:216). El instrumento se basa en la vivencia experimentada y expresada por cada sujeto. No obstante, y de acuerdo con los expertos que han elaborado el instrumento, la interpretación de los resultados ha de ser cautelosa y comprobada con otro tipo de medidas, ya que las respuestas pueden estar distorsionadas debido "al sesgo de la deseabilidad social". En este momento, únicamente pretendemos hacer una recogida de necesidades educativas a través de las diferentes dimensiones ofrecidas por el cuestionario, pudiendo servir para detectar qué sujetos deben ser diagnosticados individualmente, al mismo tiempo que ofrece una visión global del ambiente del aula.

La versión utilizada consta de 20 ítems reagrupados en cuatro dimensiones -5 ítems por cada dimensión- a puntuar mediante cuatro grados de valoración $(0$, si la conducta formulada en cada ítem no sucede nunca; 1, Algunas veces; 2, Con frecuencia, y 3 , Siempre), permitiendo un análisis de los resultados de carácter cuantitativo. Los 
resultados obtenidos en la prueba sirven de indicadores de lo que se pretenden medir desde las cuatro dimensiones establecidas: 1. Conciencia y control emocional; 2. Autoestima; 3. Habilidades socioemocionales; 4. Habilidades de vida y bienestar. A continuación definimos muy brevemente cada una de las dimensiones integradas en el cuestionario, según Bisquerra (2003) y Renom (2003):

1. Conciencia y control emocional: Capacidad para reconocer los propios sentimientos, relacionarlos con los estímulos que los provocan y utilizarlos de manera correcta. Tener conciencia de las propias emociones es la competencia emocional fundamental sobre la cual se constituyen las demás. Las emociones son impulsos que llevan a la acción, de aquí la importancia de una educación emocional apropiada a la edad de cada sujeto.

2. Autoestima: Valoración y estima que uno siente hacia sí mismo. La autoestima afecta a las relaciones que se establecen con otras personas, es decir, con todos aquellos con los que nos comunicamos y convivimos. La autoestima es una necesidad muy importante para el ser humano y es indispensable para el desarrollo psicológico de las personas. En definitiva, una autoestima positiva constituye un factor relevante en el ajuste emocional.

3. Habilidades socioemocionales: Tales habilidades constituyen un conjunto de competencias que facilitan las relaciones interpersonales. Para favorecer un clima positivo en la escuela, familia o en otros contextos, el sujeto ha de desarrollar habilidades, principalmente, de comunicación y diálogo con sus semejantes.

4. Habilidades de vida y bienestar subjetivo: Estas habilidades se conciben como competencias necesarias para la vida. Incluye la experiencia de bienestar subjetivo, como son las emociones agradables, en aquellas actuaciones que se realizan diariamente, ya sea en la escuela, la familia o en el tiempo libre.

En la práctica educativa el CEE-R está orientado a servir de ayuda tanto a los tutores como al propio alumnado. Por una parte, se presta conocer de manera general el estado emocional de cada sujeto en particular y del grupo clase en general. Por otra, contribuye a conocer las emociones que cada sujeto siente habitualmente y las reacciones que las provocan. También colabora a reflexionar sobre aquellos aspectos que influyen en sus propias emociones. Los resultados favorecen la planificación de actuaciones educativas emocionales de acuerdo con las necesidades detectadas.

\section{Procedimiento}

El cuestionario ha sido aplicado por los tutores de cada grupo en el aula habitual a lo largo del mes de diciembre y enero del curso académico 2007/2008. Previamente a la aplicación, y con el objeto de obtener la colaboración necesaria por parte del personal del centro educativo, se contactó con la Dirección de los centros 
seleccionados y con los tutores de $6^{\circ}$ Curso de Educación Primaria, que voluntariamente accedían a colaborar en el estudio.

Para conseguir una correcta aplicación del instrumento, se proporcionó información a los tutores acerca de las normas de aplicación, características del cuestionario y finalidad de la prueba. Asimismo, los alumnos, antes de cumplimentar el cuestionario, recibieron las explicaciones que, a juicio del tutor, necesitaban para tener una buena comprensión del contenido de cada ítem, al que debían de responder con una única respuesta a elegir entre cuatro alternativas.

\section{RESULTADOS}

A partir de los objetivos del estudio y dados los supuestos metodológicos, se ha considerado pertinente realizar análisis descriptivos (medias y desviaciones típicas) por Centros, grupos de sujetos, género y dimensiones ( $A=$ Control emocional; $B=$ Autoestima; $C=$ Habilidades socioemocionales; $D=$ Habilidades para la vida). También hemos recurrido al análisis de varianza para comprobar la existencia de diferencias estadísticamente significativas por grupo y género en cada una de las dimensiones.

Para una mejor comprensión de los resultados expuestos en las tablas 1, 2, 3 y 4 , y en la figura 2, conviene tener presente la amplitud de la escala. La puntuación total de cada una de las dimensiones recogidas en el cuestionario va de 0 a 15 puntos. Las respuestas proporcionadas por los sujetos del estudio están comprendidas entre valores mínimos de 4 y máximos de 15.

Tabla 1. Medias y Desviaciones Típicas por Centros, Grupos y Dimensiones

\begin{tabular}{|c|c|c|c|c|c|c|}
\hline \multirow{3}{*}{$\begin{array}{ll}\text { CENTROS } \\
\text { CENTRO I } \\
\text { DIMENSIONES } \\
\end{array}$} & \multicolumn{6}{|c|}{ GRUPOS } \\
\hline & \multicolumn{3}{|c|}{ ESPAÑOLES } & \multicolumn{3}{|c|}{ INMIGRANTES } \\
\hline & $\mathbf{N}^{\mathbf{0}}$ & $\mathbf{X}$ & DT & $\mathbf{N}^{\mathbf{0}}$ & $\mathbf{X}$ & DT \\
\hline A. Control emocional & 35 & 12.40 & 2.14 & 8 & 11.88 & 3.18 \\
\hline B. Autoestima & 35 & 11.66 & 2.86 & 8 & 10.0 & 3.62 \\
\hline C. Habilidades socioemocionales & 35 & 9.71 & 1.77 & 8 & 8.88 & 1.24 \\
\hline D. Habilidades para la vida & 35 & 12.46 & 2.38 & 8 & 11.13 & 4.88 \\
\hline CENTRO 2 & \multicolumn{3}{|c|}{ ESPAÑOLES } & \multicolumn{3}{|c|}{ INMIGRANTES } \\
\hline DIMENSIONES & $\mathbf{N}^{\circ}$ & $\mathbf{X}$ & DT & $\mathbf{N}^{\circ}$ & $\mathbf{X}$ & DT \\
\hline A. Control emocional & 9 & 13.00 & 2.23 & 14 & 11.14 & 2.53 \\
\hline B. Autoestima & 9 & 10.67 & 3.35 & 14 & 11.43 & 2.95 \\
\hline C. Habilidades socioemocionales & 9 & 11.00 & 1.93 & 14 & 11.79 & 2.54 \\
\hline D. Habilidades para la vida & 9 & 8.44 & 2.65 & 14 & 10.21 & 2.77 \\
\hline CENTRO 3 & \multicolumn{3}{|c|}{ ESPAÑOLES } & \multicolumn{3}{|c|}{ INMIGRANTES } \\
\hline DIMENSIONES & $\mathbf{N}^{0}$ & $\mathbf{X}$ & $\mathbf{N}^{\circ}$ & $\mathbf{X}$ & $\mathbf{N}^{\mathbf{0}}$ & $\mathbf{X}$ \\
\hline A. Control emocional & 18 & 13.17 & 2.89 & 2 & 12.00 & 4.24 \\
\hline B. Autoestima & 18 & 12.17 & 2.43 & 2 & 11.00 & 4.24 \\
\hline C. Habilidades socioemocionales & 18 & 11.06 & 1.39 & 2 & 11.50 & 0.70 \\
\hline D. Habilidades para la vida & 18 & 9.78 & 2.15 & 2 & 13.00 & 1.41 \\
\hline CENTRO 4 & \multicolumn{3}{|c|}{ ESPAÑOLES } & \multicolumn{3}{|c|}{ INMIGRANTES } \\
\hline DIMENSIONES & $\mathbf{N}^{\mathbf{0}}$ & $\mathbf{X}$ & $\mathbf{N}^{\mathbf{o}}$ & $\mathbf{X}$ & $\mathbf{N}^{\circ}$ & $\mathbf{X}$ \\
\hline A. Control emocional & 16 & 13.94 & 2.08 & 2 & 11.50 & 4.95 \\
\hline B. Autoestima & 16 & 12.50 & 1.54 & 2 & 12.00 & 4.24 \\
\hline C. Habilidades socioemocionales & 16 & 10.13 & 2.41 & 2 & 9.00 & 1.41 \\
\hline D. Habilidades para la vida & 16 & 8.88 & 2.41 & 2 & 10.00 & 4.24 \\
\hline
\end{tabular}


En cuanto al análisis descriptivo de las cuatro dimensiones y en la totalidad de los centros, las puntuaciones medias se encuentran dentro de los parámetros normales (ver tabla 1). Sin embargo, se debe destacar las altas puntuaciones alcanzadas en la primera y segunda dimensión Control emocional y Autoestima en los cuatro Centros y en los dos grupos. No obstante, comparando estas mismas medias observamos que las puntuaciones más altas pertenecen al grupo "españoles" en la totalidad de los Centros. Siguiendo en las mismas dimensiones $A$ y $B$, y con referencia a la desviación típica, éstas son más elevadas en el grupo "inmigrantes", es decir, su dispersión es mayor; mientras que los valores de las desviaciones típicas del grupo "españoles", excepto en la dimensión $B$ del Centro 2, están más próximos a los valores de la media aritmética correspondiente. Respecto de la dimensión Habilidades socioemocionales, ambos grupos alcanzan valores medios semejantes, y los valores de la desviación típica reflejan una dispersión menor en general. Finalmente, en la dimensión Habilidades para la vida, las medias son más elevadas en el grupo "inmigrantes", con excepción del Centro 1. En esta dimensión, la desviación típica obtenida por el grupo "inmigrantes", en los Centros 1 y 4, alcanza valores de dispersión elevados (4.88 y 4.24 respectivamente)

Figura 2. Valor medio total por grupo de sujetos y dimensiones

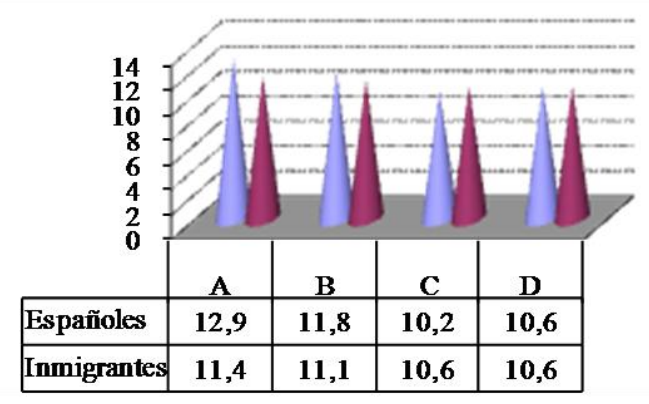

Si tenemos en cuenta la media aritmética total obtenidas por cada grupo (ver figura 2), podemos observar que las puntuaciones promedio están dentro de los parámetros de normalidad. La puntuación más alta se encuentra en la dimensión Control emocional $(\mathrm{X}=12.9)$ obtenida por el grupo "españoles"; mientras que los valores medios de las dimensiones $B, C$ y $D$ son muy semejantes en ambos grupos.

Tabla 2. Puntuaciones medias y desviaciones típicas por grupo de sujetos, género y dimensiones

\begin{tabular}{|c|c|c|c|c|c|c|c|c|c|c|c|c|}
\hline \multirow{3}{*}{ DIMENSIONES } & \multicolumn{6}{|c|}{ GRUPO “ESPAÑOLES" } & \multicolumn{6}{|c|}{ GRUPO “INMIGRANTES” } \\
\hline & \multicolumn{3}{|c|}{ Varones } & \multicolumn{3}{|c|}{ Mujeres } & \multicolumn{3}{|c|}{ Varones } & \multicolumn{3}{|c|}{ Mujeres } \\
\hline & $\mathbf{N}^{\circ}$ & $\mathbf{X}$ & DT & $\mathbf{N}^{\circ}$ & $\mathbf{X}$ & DT & $\mathbf{N}^{\circ}$ & $\mathbf{X}$ & DT & $\mathbf{N}^{\circ}$ & $\mathbf{x}$ & DT \\
\hline A. Control emocional & 38 & 12.95 & 2.20 & 40 & 12.98 & 2.53 & 12 & 11.25 & 2.92 & 14 & 11.64 & 2.84 \\
\hline B. Autoestima & 38 & 11.42 & 2.62 & 40 & 12.23 & 2.58 & 12 & 10.08 & 3.39 & 14 & 11.79 & 2.88 \\
\hline C. Habilidades socioemocionales & 38 & 9.89 & 2.06 & 40 & 10.60 & 1.73 & 12 & 9.75 & 2.05 & 14 & 11.43 & 2.53 \\
\hline D. Habilidades para la vida & 38 & 10.21 & 2.96 & 40 & 11.05 & 2.77 & 12 & 10.92 & 4.18 & 14 & 10.50 & 2.90 \\
\hline
\end{tabular}


Los resultados obtenidos por grupos (españoles e inmigrantes) y por género (varones y mujeres) reflejados en la tabla 2, muestran valores medios semejantes, así como también sucede lo mismo en las puntuaciones obtenidas en las desviaciones típicas. Únicamente señalar que en la dimensión Habilidades para la vida la desviación típica del grupo de varones inmigrantes (4.18) la consideramos elevada respecto de su media y en comparación con el resto de las puntuaciones típicas obtenidas en las restantes dimensiones.

Tabla 3. ANOVA. Grupos de sujetos y dimensiones del estudio

\begin{tabular}{lcccc}
\hline & \multicolumn{2}{c}{ GRUPO “ESPAÑOLES" } & \multicolumn{2}{c}{ GRUPO “INMIGRANTES" } \\
\cline { 2 - 5 } & F & sig. & F & sig. \\
\hline A. Control emocional & .003 & .959 & .120 & .732 \\
B. Autoestima & 1.854 & .177 & 1.910 & .180 \\
C. Habilidades socioemocionales & 2.677 & .106 & 3.369 & .079 \\
D. Habilidades para la vida & 1.672 & .200 & 0.089 & .768 \\
\hline
\end{tabular}

Para analizar el efecto de la variable grupo (españoles e inmigrantes), respecto a determinar si existen diferencias estadísticamente significativas en las dimensiones del estudio, se ha realizado un ANOVA (prueba F de Fisher) de los datos correspondientes. Los resultados obtenidos no arrojan diferencias significativas en ningún caso (ver tabla 3 ).

Tabla 4. ANOVA. Género y dimensiones del estudio

\begin{tabular}{lcccc}
\hline & \multicolumn{2}{c}{ GRUPO “VARONES" } & \multicolumn{2}{c}{ GRUPO “MUJERES" } \\
\cline { 2 - 5 } & F & sig. & F & sig. \\
\hline A. Control emocional & 4.601 & .037 & 2.687 & .107 \\
B. Autoestima & 2.050 & .159 & 0.282 & .598 \\
C. Habilidades socioemocionales & 0.045 & .833 & 1.842 & .181 \\
D. Habilidades para la vida & 0.422 & .519 & 0.398 & .531 \\
\hline
\end{tabular}

Asimismo, se pretende determinar mediante el análisis de varianza si existen diferencias significativas en las dimensiones tomadas para el estudio en función del género (varones y mujeres). El único efecto significativo encontrado ha sido en el grupo "varones" en la dimensión Control emocional $[\mathrm{F}(4.601)=0.037 ; \mathrm{p}<0.05]$ (ver tabla 4).

\section{DISCUSION}

Plantear un estudio donde la recogida de información se lleva a cabo en el propio centro y de los propios alumnos, ha supuesto un acercamiento a la realidad en la que se encuentran inmersos tantos los sujetos de estudio, de culturas diversas, como los profesores de estos mismos sujetos. Con ello, también se ha pretendido fomentar en los tutores el interés por el tema y la necesidad de formación, así como la de ampliar los horizontes en el ámbito de la educación emocional. 
Al confrontar los resultados obtenidos en el estudio con cada uno de los objetivos propuestos en la investigación, surgen las siguientes conclusiones:

Del análisis de los datos recogidos a través del instrumento "Educación emocional", se observan puntuaciones bajas y muy bajas en un número reducido de alumnos, en ambos grupos y en la totalidad de los Centros. Estos sujetos supuestamente identificados con necesidades emocionales, serían orientados hacia una evaluación diagnóstica, para comprobar de manera rigurosa y precisa sus carencias emocionales. Por otro lado, y desde el punto de vista de cada centro y dimensión, la desviación típica más elevada, debido precisamente a tales puntuaciones, se encuentran, en general, en el grupo "inmigrantes".

Si analizamos los valores de la media y desviación típica por grupo-clase y dimensiones, observamos lo siguiente:

Centro 1: Los alumnos "españoles" en las dimensiones Control emocional, Autoestima y Habilidades para la vida, obtienen puntuaciones medias altas, mientras que resultan más próximas a la media del grupo las puntuaciones de la dimensión Habilidades socioculturales. Las desviaciones típicas correspondientes a tales dimensiones tienen valores de dispersión aceptable si se comparan con su media correspondiente. En el mismo centro y observando los valores del grupo "inmigrantes" las puntuaciones medias son más bajas y la dispersión es mayor.

Centro 2: Tanto las puntuaciones medias como las desviaciones típicas, en las cuatro dimensiones y en los dos grupos, son semejantes. No obstante, la puntuación media más elevada se encuentra en la dimensión Control emocional en el grupo "españoles". Y, es en este centro donde se encuentra la puntuación media más baja del estudio en la dimensión Habilidades para la vida en el grupo "españoles”, resultado más elevadas las del grupo "inmigrantes".

Centro 3: Lo más destacable está en las puntuaciones desviaciones típicas (4.2 y 4.1) obtenidas por el grupo "inmigrantes" en las dimensiones Control emocional y Autoestima, por ser éstas elevadas en comparación con su media respectiva y con las puntuaciones obtenida en las mismas dimensiones por el grupo "españoles".

Centro 4: Al igual que en el grupo anterior, lo que más despunta son las desviaciones típicas $(4.95 ; 4.24 ; 4.24)$ de las dimensiones Control emocional, Autoestima y Habilidades para la vida en el grupo "inmigrantes", por su amplia dispersión respecto de su media aritmética y en comparación con las puntuaciones en las mismas dimensiones del grupo "españoles".

Una revisión pormenorizada de las puntuaciones obtenidas por los sujetos de cada grupo (españoles e inmigrantes) y desde la variable género (varones y mujeres) en cada dimensión, nos permite concluir que las diferencias, en cuanto a necesidades emocionales, no llegan a ser estadísticamente significativas. Únicamente se encuentran diferencias significativas en el grupo "varones" en la dimensión Control emocional. Esta 
capacidad supone reconocer los propios sentimientos, relacionarlos con los estímulos que los provocan y utilizarlos de manera correcta. Ello supondría un planteamiento educativo en la dimensión citada, puesto que el control de las propias emociones se considera como la competencia fundamental sobre la cual se constituyen las demás capacidades.

Por último, indicar que este trabajo pone de manifiesto la conveniencia de educar las emociones de los estudiantes, tanto españoles como inmigrantes, así como a desarrollar una autoestima positiva de sí mismo en los sujetos con puntuaciones bajas, puesto que afecta a las relaciones que se establecen con otras personas. Así también, desarrollar habilidades socioemocionales que faciliten principalmente las relaciones interpersonales. Del mismo modo, las competencias necesarias para la vida como son las emociones agradables en las acciones diarias y en los contextos en los que los sujetos están inmersos. Una adecuada educación en el ámbito de las emociones colabora a favorecer las relaciones personales en distintos contextos y a potenciar la convivencia social.

Finalmente, queremos señalar que somos conscientes de la necesidad de seguir profundizando en los hallazgos referidos al tema abordado en este trabajo. Pensamos que esta investigación, primera que se realiza en Asturias específicamente con sujetos de diversos países y culturas sobre "educación emocional", en el curso $6^{\circ}$ de Educación Primaria, puede aportar sugerencias para reflexionar sobre el desarrollo de las competencias emocionales, ya que se pueden aplicar a una multiplicidad de situaciones (Bisquerra, 2003).

\section{REFERENCIAS}

Alvarez, M. (2001). Diseño y evaluación de programas de educación emocional. Barcelona: CISSPRAXIS.

Bisquerra, R. (2003). Educación emocional y competencias básicas para la vida. Revista de Investigación Educativa, (21)1, 7-43.

Collel, J. y Escudé, C. (2003). L'educació emocional. Traç. Revista dels mestres de la Garrotxa, $37,8-10$.

Dueñas, M.L. (2002). Importancia de la inteligencia emocional: un nuevo reto para la orientación educativa. EDUCACIÓN XXI, 5, 77-96.

Fernández, M. y Terrén, E. (2008). Presentación. De inmigrantes a minorías: temas y problemas de la multiculturalidad. Revista de Educación, 345, 15-21.

Delors, J (1998). Informe Delors. La educación encierra un tesoro. Madrid: UNESCO-Santillana. Ley Orgánica 2/2006, de 3 de mayo de Educación.

Moreno, J. (2006). Aprender a convivir en una escuela intercultural. En E. Soriano (Coord.), Convivencia y mediación intercultural (pp. 11-17). Almería: Ed. Universidad de Almería.

Kirchner, T. Torres, M. y Forns, M. (1998). Evaluación psicológica: modelos y técnicas. Barcelona: Paidós. 
Rernom, A. (2003). Educación emocional. Programa para Educación Primaria (6-12 años). Barcelona: PRAXIS.

Romero, J.B. y Pérez, H.M. (2008). Interculturalidad y escuelas democráticas en la sociedad actual. En I Jornadas Interculturales. VI Jornadas sobre Diagnóstico y Orientación. Jaén, 21-23 de Febrero.

Recibido: 2 de enero de 2009

Recepción Modificaciones: 5 de abril de 2009

Aceptado: 25 de mayo de 2009 